Moulay Alaoui-Jamali Sankar Mitra

\section{Les mécanismes de réparation de I'ADN : des cibles potentielles en pharmacologie du cancer}

La résistance à la chimiothérapie est fréquente en cancérologie. Une tumeur peut être intrinsèquement résistante aux médicaments ou peut développer, au cours du traitement, différents mécanismes de résistance. Ainsi, les cellules peuvent neutraliser les lésions d'ADN provoquées par les agents génotoxiques (alkylants, analogues du cis-platine) grâce à des mécanismes de réparation aboutissant à l'excision d'adduits* formés au niveau d'une base, l'excision de base, ou l'excision de nucléotide. La réparation de l'ADN par la $\mathrm{O}^{6}$-alkylguanine-ADN-méthyltransférase (MGMT) est le mécanisme le mieux caracterisé; l'inhibition de la MGMT dans les cellules tumorales augmente la cytotoxicité des chloro-nitrosourées, alors que son transfert dans des cellules de la moelle osseuse protège celles-ci contre la myélosuppression induite par ces médicaments. On est moins avancé dans la compréhension des mécanismes de l'altération de la réparation par excision de nucléotides dans les cellules tumorales résistantes aux anti-cancéreux, mais les connexions entre la réparation, la transcription et le cycle cellulaire offrent des cibles multiples pour moduler ou cibler la réparation dans le contexte thérapeutique.

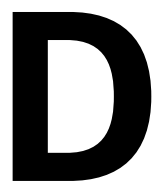

urant la dernière décennie, d'énormes efforts ont apporté l'espoir d'améliorer le traitement des maladies cancéreuses. De nom-

M. Alaoui-Jamali : professeur chercheur. Institut Lady Davis de recherches médicales de l'hôpital général juif, départements de médecine et d'oncologie, faculté de médecine, université McGill, 3755, Côte-Sainte-Catherine, Montréal, Québec, Canada. S. Mitra: professeur titulaire. Center for Molecular Sciences, the University of Texas Medical Branch at Galveston, 301 University boulevard, Galves- breuses alternatives de traitements ont été découvertes incluant des agents cytotoxiques nouveaux et des

* Pour le mot anglais adduct, radical lié de manière covalente à l'ADN. combinaisons d'agents multiples, des analogues d'hormones, et des agents biologiques. Parmi ces différentes approches thérapeutiques, la chimiothérapie demeure un traitement de choix pour les maladies avancées. Cependant, il est très fréquent que son efficacité soit provisoire et que la maladie réapparaisse après des périodes plus ou moins longues. Les raisons de ces échecs thérapeutiques 


\section{RÉFÉRENCES}

1. Lepage P, Gros P. La glycoprotéine P: de la résistance croisée aux médicaments au transport des lipides biliaires. médecine/ sciences $1995 ; 11$ : 357-66.

2. Cole SPC, Bhardwaj G, Gerlach JH, Mackie JE, Grant CE, Almquist KC, Stewart AJ, Kurz EU, Duncan AMV, Deeley RG. Overexpression of a transporter gene in a multidrug-resistant human lung cancer cell line. Science 1992; 258: 1650-3.

3. Scheffer GL, Wijngaard PLJ, Flens MJ, Izquierdo MA, Slovak ML, Pinedo HM, Meijer CJLM, Clevers HC, and Scheper RJ. The drug resistance-related protein LRP is the human major vault protein. Nature Med $1995 ; 1: 578-82$.

4. Batist G, Schecter RL, Alaoui Jamali MA. The glutathione system and drug resistance. In : Richard Schilsky ed. Principles of Cancer Drug Pharmacology. New York : Marcel Dekker, 1996 (sous presse).

5. Gupta RS, Ross WE. Etoposide (VP16), teniposide (VM26), and related epipodophyllotoxin derivatives. In : drug resistance in mammalian cells, vol. 2. Boca Raton, FL: CRC Press, 1989 : 89-107.

6. Teicher BA, Herman TS, Holden SA, Wang Y, Pfeffer MR, Crawford JW, Frei III E. Tumor resistance to alkylating agents conferred by mechanisms operative only in vivo. Science 1990; 247: 1457-61.

7. Sarasin A. Les gènes humains de la réparation de l'ADN. médecine/sciences 1994; 10 : 43-54.

8. Sancar A. Mechanims of DNA excision repair. Science 1994; 266: 1954-6.

9. Pegg AE, Byers TL. Repair of DNA containing $\mathrm{O}^{6}$-alkylguanine. FASEB J 1992; $6: 2302-10$

10. Dumenco LL, Allay E, Norton K, Gerson SL. The prevention of thymic lymphomas in transgenic mice by human $\mathrm{O}^{6}$-alkylguanineDNA alkyltransferase. Science 1993; 259: 219-22.

11. Mitra S, Kaina B. Regulation of alkylation damage in mammalian genomes. Prog Nucleic Acids Res Mol Biol 1993; 44: 109-42.

12. Fritz G, Tano K, Mitra S, Kaina B. Inducibility of the DNA repair gene encoding $\mathrm{O}^{6}$-methylguanine-DNA-methyltransferase in mammalian cells by DNA-damaging treatments. Mol Cell Biol 1991; 11: 4660.

13. Citron, M, Decker R, Chen, S, Shneider S, Graver M, Kleynerman L, Kahn LB, White A, Schoenhaus M, Yarosh D. $\mathrm{O}^{6}$-methylguanine-DNA-methyltransferase in human normal and tumor tissue from brain, lung and ovary. Cancer Res 1991 ; 51 : 4131-4.

14. Dolan, ME, Mitchell B, Mummelt C, Moschel RC, Pegg AE. Effects of $\mathrm{O}^{6}$-benzylguanine analogues on sensitivity of human tumor cells to the cytotoxic effects of alkylating agents. Cancer Res $1991 ; 51: 3367$ 72 .

$m / s n^{\circ} 6-7$, vol. 12 , juin-juillet 96

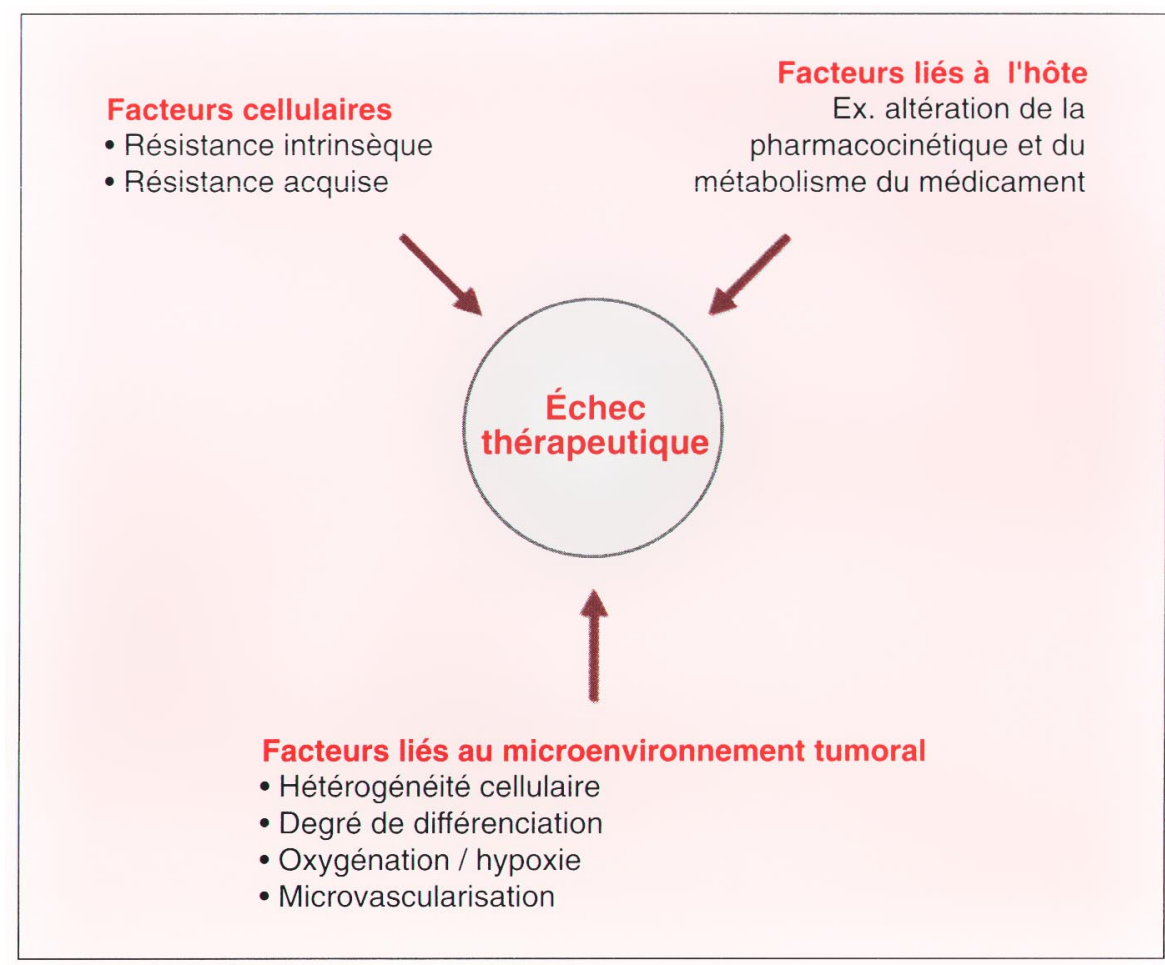

Figure 1. Principaux mécanismes responsables des échecs thérapeutiques en clinique cancérologique.

sont multiples et complexes et peuvent être liées, soit à l'hôte (le médicament est mal toléré, son métabolisme ou sa pharmacocinétique sont modifiés, etc.), soit à la tumeur et à son micro-environnement (figure 1). Une tumeur peut être, pour des raisons encore peu élucidées, intrinsèquement résistante aux médicaments (c'est le cas fréquent du cancer du côlon et du cancer du poumon à l'exception de celui à petites cellules), ou peut développer, au cours du traitement, différents mécanismes de résistance (résistance acquise). Le mécanisme le plus étudié de la résistance acquise est la surexpression de la glycoprotéine membranaire P170. Cette protéine est capable de refouler certains médicaments, en particulier les anthracyclines, du compartiment intracellulaire vers le compartiment extracellulaire [1]. D'autres mécanismes de résistance acquise incluent l'expression d'autres protéines de transport membranaire telles que la MRP (multidrug resistance-associated protein) [2] et la LRP (lung resistancerelated protein) [3], une diminution du métabolisme intracellulaire du médicament ou son inactivation par des enzymes de détoxification [4], une altération de la cible cellulaire du médicament [5], et une augmentation des capacités de réparation. Tous ces mécanismes permettent à la cellule tumorale d'échapper aux effets toxiques des médicaments et/ou à la mort cellulaire programmée (apoptose) attribuée à de nombreux agents anticancéreux. Le micro-environnement tumoral peut également jouer un rôle dans la résistance. Ainsi l'hétérogénéité des cellules tumorales, la création de conditions hypoxiques, la présence ou l'absence d'interactions entre les cellules, ou des changements de la microvascularisation tumorale peuvent contribuer à la résistance [6]. De nombreux travaux y compris les nôtres ont démontré que différents mécanismes de résistance peuvent coexister au sein d'une même tumeur.

De nombreux médicaments anticancéreux, en particulier les agents alkylants et les analogues du cis-platine, exercent leurs effets cytotoxiques en formant des adduits et des liaisons intra- et intercaténaires au niveau de l'ADN (DNA crosslinks). Ces lésions sont capables d'inhiber la transcrip- 


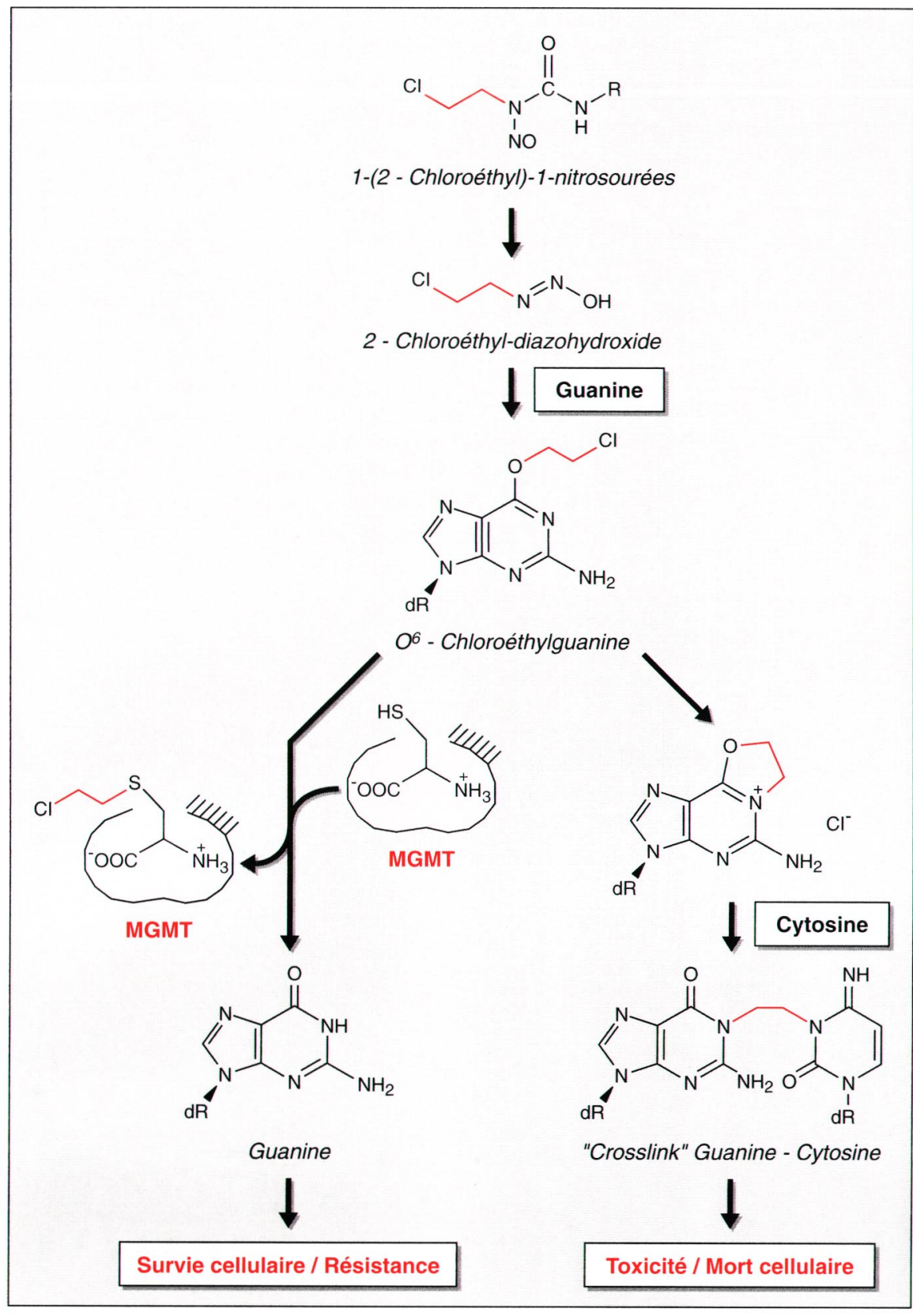

Figure 2. Mécanisme d'action de la $O^{6}$-méthyl guanine-ADN-méthyltransférase (MGMT). Les chloro-nitrosourées (CNU) sont des médicaments qui subissent une transformation chimique et non enzymatique, pour donner naissance au chloroéthyl-diazohydroxyde capable d'alkyler I'ADN (pour certains CNU il y a également formation d'isocyanates capables de carbamoyler les protéines cellulaires). Le chloroéthyl-diazohydroxyde forme un lien covalent avec l'oxygène 6 de la guanine. Deux situations sont possibles: (1) le groupement alkylant est reconnu par la MGMT qui le transfère sur son résidu cystéyl pour donner naissance à une cystéine alkylée. Dans ce cas la guanine est libérée de l'adduit et redevient normale ce qui permet à la cellule de se répliquer normalement. (2) La deuxième possibilité s'ouvre quand la MGMT est déficiente ou présente en faible quantité. Le lien covalent au niveau de l'oxygène 6 subit alors un réarrangement après déplacement de l'atome de chlore, et réagit par la suite avec la cytosine au niveau du brin opposé pour donner naissance à des ponts intercaténaires entre guanine et cytosine (d'ou le nom d'agents alkylants bifonctionnels donné aux nitrosourées). Ces ponts blo- tion, la réplication, et aboutiront à la mort cellulaire. Les cellules possèdent des mécanismes de défense assez élaborés qui sont activés par les agents génotoxiques, y compris les médicaments. En particulier, les cellules peuvent neutraliser les lésions de l'ADN par des mécanismes de réparation impliquant l'activation et/ou la régulation de nombreuses protéines et facteurs de transcription. Parallèlement, les cellules ralentissent ou arrêtent momentanément leur progression à travers le cycle cellulaire par suite des lésions de l'ADN, ce qui accordera un temps suffisant à la réparation. L'altération de l'un ou l'autre de ces mécanismes a des conséquences sur la réponse cellulaire aux médicaments.

Plusieurs mécanismes de réparation de l'ADN ont été identifiés parmi lesquels l'excision spécifique d'adduits (groupes alkylés) formés au niveau d'une base par la $\mathrm{O}^{6}$-alkylguanine-ADN-méthyltransférase, la réparation de base (excision de base unique), la réparation de nucléotide (excision de plusieurs bases sous forme de nucléotide), la réparation des mésappariements et, enfin, la réparation par recombinaison. L'objectif de cette revue n'est pas de donner une compilation de ces mécanismes de réparation qui ont été décrits en détail ailleurs [7, 8], mais de donner un aperçu de leur rôle dans la résistance aux médicaments et de leurs implications dans le traitement du cancer.

\section{La $O^{6}$-alkylguanine-ADN- méthyltransférase et son rôle dans la résistance}

Un certain nombre de médicaments anticancéreux, en particulier les analogues des chloro-nitrosourées $(C N U)$ tels que le 1,3-bis(2-chloroéthyl)-1-nitrosourée (BCNU), 1-(2-chloroéthyl)-3-cyclohexyl-1-nitrosourée (CCNU), 1-(2-chloroéthyl)-3-méthylcyclohexyl-1-nitrosourée (méthyle-CCNU), ou 1-(2-chloroéthyl)-1nitroso-3-(2-hydroxyéthyl) urée (HECNU), utilisés dans le traitement de plusieurs cancers (en particulier les gliomes, lymphomes, mélanomes, myélomes multiples, et les cancers du tractus digestif), exercent leurs effets cytotoxiques principalement par formation de monoadduits au niveau de la guanine $\left(\mathrm{O}^{6}\right.$-chloroéthylguanine), 
suivie d'un réarrangement et de la formation de liaisons intercaténaires (crosslinks) (figure 2).

\section{RÉFÉRENCES}

15. Moschel RC, McDougall MG, Dolan ME, Stine L, Pegg AE. Structural features of substituted purine derivatives compatible with depletion of human $\mathrm{O}^{6}$-alkvlguanine-DNA alkyltransferase. J Med Chem 1992; 35 : 4486-91.

16. Micetich KC, Futscher BW, Koch D, Fisher RI, Erickson LC. A phase I study of streptozotocin and BCNU sequenced administration in patients with advanced cancer. I Natl Cancer Inst 1992; 84 : 256-60.

17. Allay JA, Dumenco LL, Koc ON, Liu L, Gerson SL. Retroviral transduction and expression of the human alkyltransferase cDNA provides nitrosourea resistance to hematopoietic cells. Blood 1995 ; 85 : 3342-51.

18. Moritz T, Mackay W, Glassner BJ, Williams DA, Samson L. Retrovirus-mediated expression of a DNA repair protein in bone marrow protects hematopoietic cells from nitrosourea-induced toxicity in vitro and in vivo. Cancer Res 1995; 55 : 2608-14.

19. Schaeffer L, Egly J. BTF2/TFIIH, un facteur de transcription et réparation impliqué dans des maladies de la réparation de l'ADN. médecine/sciences 1994; 10 : 973-8.

20. Roy R, Bergmann E, Egly J. TFIIH (BTF2), à l'interface de trois processus cellulaires : transcription, réparation et cycle cellulaire. médecine/sciences 1995; 11 : 879-82.

21. Lai G, Ozol RF, Smyth JF, Young RC, Hamilton TC. Enhanced DNA repair and resistance to cisplatinum in human ovarian cancer. Biochem Pharmacol 1988; 37 : 4597600 .

22. Dabholkar M, Bostick-Bruton F, Weber C, Bohr V, Egwuagu C, Reed E. ERCC1 and ERCC2 expression in malignant tissues from ovarian cancer patients. J Natl Cancer Inst 1992; 84: 1512-7.

23. Geleziunas R, McQuillan A, Malapetsa A, Hutchinson M, Kopriva D, Wainberg MA Hiscott J, Bramson J, Panasci L. Increases DNA synthesis and repair-enzyme expression in lymphocytes from patients with chronic lymphocytic leukemia resistant to nitrogen mustards. J Natl Cancer Inst 1992; 84: 1512-7.

24. Yen L, Christopoulopoulos R, Panasci L, Batist G, Rabindra R, Mitra S, Alaoui-Jamali MA. Enhanced host cell reactivation capacity and expression of DNA repair genes in human breast cancer cells resistant to bifunctional alkylating agents. Mutat Res 1995; 337: 179-89.

25. Nie ZR, Paterson J, Alpert L, Tsao MS, Viallet J, Alaoui-Jamali MA. Elevated DNA repair capacity is associated with intrinsic resistance of lung cancer to chemotherapy. Cancer Res 1995 ; 55 : 4760-4.

26. Fraval HN, Rawlings C, Roberts IJ. Increased sensitivity of UV-repair-deficient human cells to DNA bound platinum products which unlike thymine dimers are not recognized by the endonuclease extracted from Micrococcus luteus. Mutat Res 1978; 51 : 12132.

$m / s n^{\circ} 6-7$, vol. 12 , juin-juillet 96

\section{La protéine MGMT}

La protéine MGMT ( $\mathrm{O}^{6}$-méthylguanine-ADN-méthyltransférase) permet le transfert stoïchiométrique direct et en une seule étape du groupement alkylé formé au niveau d'une base d'ADN, principalement au niveau de l'oxygène 6 de la guanine, sur son résidu cystéyl (figure 2). Cela permet la restauration de la structure normale de la base et prévient donc la formation d'un monoadduit, précurseur des liaisons intercaténaires nécessaires à l'effet cytotoxique de ces médicaments. Le complexe S-alkylcystéine formé est stable et la protéine devient inactive et n'est pas régénérée par la suite d'où l'appellation "enzyme suicide » [9]. La mutagénicité élevée et la tendance de la guanine alkylée à s'apparier avec la thymine (au lieu de la cytosine, ce qui donne lieu à des mutations par transition G:C/A:T ou $\mathrm{T}: \mathrm{A} / \mathrm{G}: \mathrm{C})$ illustrent l'importance de la MGMT dans la neutralisation des mutations avant que la cellule réplique son ADN (d'où son rôle crucial en cancérogenèse chimique). La surexpression de la MGMT protège les cellules normales des effets mutagènes et cancérigènes de nombreux agents alkylants, y compris les CNU, in vitro et in vivo sur des souris transgéniques $[10,11]$.

Le gène codant pour la MGMT a été caractérisé dans plusieurs espèces y compris l'homme [11]. Malgré certaines divergences interespèces, la séquence avoisinant le résidu cystéyl qui accepte le groupement alkyl est bien conservée. La protéine est liée en partie à la chromatine et semble avoir une composante de reconnaissance de l'ADN peu spécifique et à faible affinité, ainsi qu'une autre plus spécifique des lésions de l'ADN et d'affinité élevée. Elle est inductible par les agents alkylants, les radiations ionisantes et ultra-violettes [11, 12]. Outre son affinité élevée pour la guanine alkylée, la MGMT humaine semble aussi agir contre les groupements méthyles alkylés au niveau de l'oxygène 4 de la thymine, alors que cette affinité est très faible pour les groupements éthyle alkylés [9].

La régulation de la MGMT se fait essentiellement au niveau transcriptionnel mais d'autres mécanismes de régulation, telle que la stabilité des ARNm, semblent être impliqués [11]. Chez l'homme, la MGMT est majoritairement synthétisée dans le foie, tandis que le cerveau, les lymphocytes et les cellules de la moelle osseuse sont les tissus qui en produisent le moins [13]. L'activité enzymatique dans les tissus et les cellules cancéreuses varie énormément et son niveau semble déterminer le degré de résistance aux médicaments de la classe des CNU. Une activité élevée de la MGMT a été détectée dans de nombreuses cellules et tumeurs résistantes à ces médicaments in vitro et in vivo [9]. Le mécanisme de cette augmentation reste encore inconnu mais semble être indépendant de l'amplification de gènes [11].

\section{Amélioration de la tolérance aux chloro-nitrosourées}

Deux approches ont été utilisées pour manipuler la MGMT dans le but de sensibiliser les tumeurs résistantes aux médicaments de la classe des chloro-nitrosourées et d'améliorer la tolérance à ces médicaments.

- La première est fondée sur l'inhibition directe de la protéine dans les cellules tumorales qui en produisent une grande quantité. Les modulateurs les plus utilisés pour inactiver la MGMT sont la $\mathrm{O}^{6}$-méthylguanine libre, $\mathrm{O}^{6}$-benzylguanine $(\mathrm{BG})$ et $\mathrm{O}^{6}$-benzyl-2'-désoxyguanosine ( $\left.\mathrm{dBG}\right)$ $[14,15]$. La MGMT peut également être inhibée par l'utilisation d'agents méthylants de l'ADN tels que la streptozotocine. Cette dernière induit l'alkylation de l'oxygène 6 de la guanine, qui est un substrat de la MGMT, et réduit donc indirectement la quantité de MGMT disponible dans les cellules tumorales résistantes. Par conséquent, une utilisation combinée de chacun de ces inhibiteurs et des CNU augmente les effets cytotoxiques des CNU et donc leur efficacité. Cela a été démontré sur des cellules résistantes maintenues in vitro mais également in vivo sur des animaux porteurs de greffes de tumeurs humaines [9, 14]. Des 
essais cliniques de phase I ont indiqué que les modulateurs de la MGMT sont bien tolérés [16].

- La deuxième stratégie consiste à exprimer le gène de la MGMT dans les cellules de la moelle osseuse qui ont pour caractéristique une très faible concentration de cette protéine. Comme les effets toxiques secondaires des CNU sont dirigés essentiellement contre les cellules de la moelle osseuse, une augmentation de l'expression du gène de la MGMT réduit donc ces effets secondaires et permet ainsi l'utilisation de doses plus fortes de médicaments, ce qui améliore l'index thérapeutique. Les rétrovirus se sont avérés très efficaces pour transférer le gène de la MGMT et l'exprimer in vivo dans les cellules de la moelle osseuse [17]. Des études récentes ont montré que le transfert de la MGMT par les rétrovirus protège les animaux contre la myélotoxicité des CNU mais également contre la fibrose pulmonaire [18] observée secondairement chez les malades traités par ces médicaments.

\section{La réparation par excision de nucléotide dans la résistance aux médicaments}

L'excision de nucléotide (NER, nucleotide excision repair) [19] semble être un mécanisme majeur de la réparation des adduits et des liaisons inter- et intracaténaires telles que formées par de nombreux médicaments alkylants et les analogues du cis-platine. C'est un processus complexe mettant en jeu une multitude de protéines qui agissent en 3 étapes principales: reconnaissance des lésions, excision du fragment portant ces lésions, et synthèse d'un nouveau brin d'ADN normal $[7,8]$. La découverte récente d'une connexion entre cette voie de réparation, la transcription et le cycle cellulaire [20] suggère que ces différents processus sont intimement liés, et que la cellule doit coordonner ces mécanismes d'une façon adéquate afin de réparer les lésions infligées à son matériel génétique, sans compromettre sa prolifération. De nombreux laboratoires y compris le nôtre ont démontré qu'une accélération de la ré- résistance aux analogues du cis-platine et aux agents alkylants de la classe de la moutarde azotée, dans des cellules cancéreuses d'origine ovarienne [21, 22], leucémique [23], mammaire [24], et pulmonaire [25]. Cependant, le rôle de la réparation par excision de nucléotides dans la résistance in vivo reste à démontrer. Les cellules isolées de malades ayant des déficiences génétiques en réparation, en particulier le xeroderma pigmentosum groupe XPA, sont hypersensibles au cis-platine et aux agents alkylants [26]. Les cellules de rongeurs déficientes en réparation par excision de nucléotides, en particulier le groupe de complémentation 1 (représenté par les cellules UV20 et 43-3B) et le groupe 4 (représenté par les cellules UV41 et UV47) sont caractérisés par leur extrême sensibilité aux agents bifonctionnels tels que la mitomycine $\mathrm{C}$, le cis-platine et le melphalan. Les cellules du groupe 1 ont une déficience fonctionnelle du gène ERCC1 (excision repair cross complementing group 1) alors que celles du groupe 4 ont une déficience fonctionnelle du gène $E R C C 4$, suggérant que les gènes ERCC1 et ERCC4 jouent un rôle critique dans la réparation des liaisons intra-/intercaténaires formées par ces médicaments. La transfection du gène ERCC1 dans des cellules du groupe 1 confère la résistance aux agents alkylants et au cis-platine [27]. Certaines études ont démontré une corrélation entre l'expression de l'ARNm du gène ERCC1 et la résistance des tumeurs au cis-platine et aux dérivés de la moutarde azotée [22, 23]. Nous n'avons pas détecté de corrélation entre la synthèse de l'ARNm et des protéines codées par les gènes ERCC1, XPB/ERCC3 et XPD/ERCG2 dans les cellules cancéreuses du poumon résistantes au cis-platine ou des cellules du cancer du sein résistantes aux analogues de la moutarde azotée (nos résultats non publiés). Très peu de données existent à l'heure actuelle concernant les autres protéines impliquées dans la NER telles que XPA, XPC, XPG et ERCC4. L'absence d'analyse biochimique permettant de mesurer l'activité de ces protéines, la présence de certaines de ces protéines sous forme de complexe (ERCC1 forme un complexe avec ERCC4 [28]), et l'association entre la p53 et les protéines XPD/ERCC2 et XPB/ERCC3 [29] soulignent la complexité d'établir des relations étroites entre un gène/une protéine de réparation en particulier et la résistance aux médicaments. Cela est compliqué davantage par le fait que la résistance cellulaire peut également être associée à une augmentation de la réparation dans des gènes activement transcrits, sans que la réparation globale soit affectée.

Il n'existe pas, à l'heure actuelle, d'inhibiteur spécifique des étapes de reconnaissance et d'excision de nucléotides. Seuls des inhibiteurs de l'étape de synthèse sont connus. Il s'agit surtout des inhibiteurs de polymérases et des antimétabolites tels que l'hydroxyurée et l'arabinosine cytosine. Les analogues de l'aphidicoline sont les inhibiteurs de polymérases les plus utilisés et ils se sont avérés efficaces dans la sensibilisation des cellules résistantes, maintenues in vitro, aux dérivés du cis-platine. Des essais cliniques phase I avec l'aphidicoline glycinate ont démontré que cet analogue est bien toléré par les malades [30]. D’autres médicaments tels que les analogues des nucléosides (Ara-C, hydroxyurée, gemcitabine, azidothymidine, etc.) semblent interférer indirectement avec la réparation par inhibition de la synthèse des désoxynucléotides, des ligases et/ou des polymérases.

\section{Autres mécanismes de réparation pouvant jouer un rôle dans la résistance tumorale}

Outre des adduits et des lésions intra- et intercaténaires, les médicaments anticancéreux induisent des lésions secondaires au niveau de l'ADN telles que des sites apuriniques, apyrimidiniques et des coupures de brins qui semblent faire intervenir d'autres formes de réparation, en particulier l'excision de base. Il a été démontré que les lésions induites par les $\mathrm{CNU}$ et les dérivés de la moutarde azotée peuvent être réparées par les glycosylases, en particulier la MPG (pour Nméthyl purine glycosylase) [9] et de fait, une élévation de l'activité des 


\section{RÉFÉRENCES}

27. Zdzienicka MZ, Roza L, Westerveld A, Bootsma D, Simons JW. Biological and biochemical consequences of the human ERCC-1 repair gene after transfection into a repair-deficient CHO cell line. Mutat Res $1987 ; 183: 69-74$.

28. Van Vuuren AJ, Appeldoorn E, Odijk H, Yasui A, Jaspers NGJ, Bootsma D, Hoeijmakers JHJ. Evidence for a repair enzyme complex involving ERCC1 and complementing activities of ERCC4, ERCC11 and xeroderma pigmentosum group F. EMBO J 1993; 12 : 3693-701.

29. Wang XW, Yeh H, Schaeffer L, Roy R, Moncollin V, Egly JM, Wang Z, Freidberg EC, Evans MK, Taffe BG, Bohr VA, Weeda G, Hoeijmakers JHJ, Forrester K, Harris CC. p53 modulation of TFIIH-associated nucleotide excision repair activity. Nature Genet $1995 ; 10: 188-95$.

30. Sessa C, Zucchetti M, Davoli E, Califano R, Cavalli F, Frustaci S, Gumbrell L, Sulkes A, Winograd B, D'Incalci M. Phase I and clinical pharmacological evaluation of aphidicolin glycinate. J Natl Cancer Inst 1991 ; 83 : 1160-4.

31. Lautier D, Lagueux J, Thibodeau J, Menard L, Poirier G. Molecular and biochemical features of poly (ADP-ribose) metabolism. Mol Cell Biochem 1993; 122: 171-93.

32. Chu G, Chang E. Cisplatin-resistant cells express increased levels of a factor that recognizes damaged DNA. Proc Natl Acad Sci USA $1990 ; 87$ : 3324-8.

33. Gottlieb TM, Jackson SP. Protein kinases and DNA damage. Trends Biol Sci 1994; 19: 500-3.

34. Kahn A. Cycle cellulaire, cancer, sénescence et p53. médecine/sciences 1994; 10: 206.

35. Marx J. New link found between p53 and DNA repair. Science 1994; $266: 25$.

36. Reiss M, Vellucci VF, Zhou ZL. Mutant p53 tumor suppressor gene causes resistance to transforming growth factor beta 1 in murine keratinocytes. Cancer Res 1993; 53 : 899-904.

37. Nguyen KT, Liu B, Ueda K, Gottesman MM, Pastan I, Chin KV. Transactivation of the human multidrug resistance (MDR1) gene promoter by p53 mutants. Oncol Res $1994 ; 6: 71-7$.

38. Arteaga CL, Winnier AR, Poirier MC, Lopez-Larraza DM, Shawver LK, Hurd SD, Stewart SJ. p185 $5^{\mathrm{c}-e r b B-2}$ signaling enhances cisplatin-induced cytotoxicity in human breast carcinoma cells : association between an oncogenic receptor tyrosine kinase and druginduced DNA repair. Cancer Res 1994; 54 : 3758-65.

39. Henderson D, Hurley LH. Molecular struggle for transcription control. Nature Med $1995 ; 1$ : 525-7.

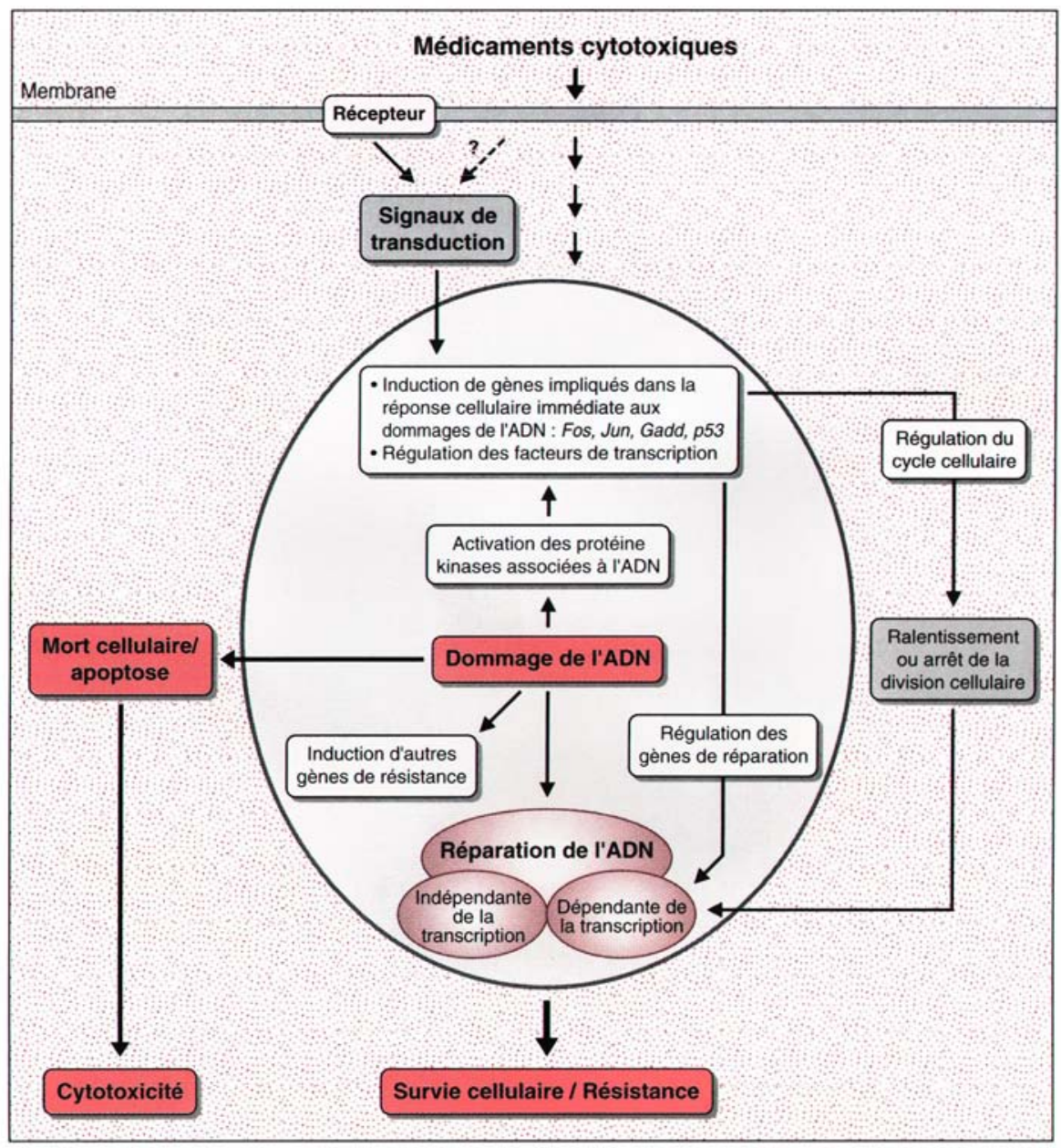

Figure 3. Réponse cellulaire aux effets toxiques des médicaments. A la suite des lésions de l'ADN provoquées par les agents génotoxiques, les cellules peuvent réagir par activation de gènes impliqués dans la réponse cellulaire immédiate aux dommages de I'ADN. Plusieurs voies de transduction sont activées par les agents génotoxiques. En outre, les dommages de l'ADN induisent des protéine kinases associées à I'ADN. Ces dernières sont capables de phosphoryler un certain nombre de gènes impliqués dans la réponse immédiate, en particulier Fos, Jun, et p53. L'induction de toute cette panoplie de protéines pourraît être responsable de: (1) l'activation des gènes impliqués dans la mort cellulaire programmée (mécanisme nécessaire à l'action cytotoxique); (2) la régulation du cycle cellulaire (arrêt des cellules en G1 pour la réparation par excision, inhibition du début de la réplication pour la réparation post-réplicative, arrêt en G2 pour la réparation des coupures doubles brins) ; (3) la réparation des lésions induites dans les gènes inactifs ou activement transcrits. Une fois que les lésions sont réparées, la cellule peut donc survivre en contournant les effets toxiques des médicaments.

glycosylases a été observée dans des cellules de leucémie lymphocytaire chronique résistantes au melphalan/chlorambucil [23]. A l'inverse de la MGMT, il n'existe pas de cellules déficientes en MPG ce qui complique l'étude du rôle de cette enzyme dans la résistance. Un autre enzyme pouvant intervenir dans l'excision de base est la PARP (pour poly (ADP-ribose) polymérase) décrite précédemment $\left(\mathrm{m} / \mathrm{s} n^{\circ} 10\right.$, vol. 11 , p. 1487) [31]. Cette enzyme, à rôles multiples, semble agir en partie dans la reconnaissance des coupures de brins induites par les médicaments et 
probablement dans le recrutement d'autres enzymes de réparation. Cependant, la majorité des données disponibles actuellement suggèrent que cette enzyme, généralement présente en abondance dans les cellules, ne semble pas jouer un rôle crucial dans la résistance aux médicaments anti-cancéreux. D'autres protéines capables de se lier aux adduits causés par le cis-platine ont été identifiées. L'une de ces protéines est absente dans certaines cellules XPE (pour xeroderma pigmentosum groupe E) mais est, à l'inverse, augmentée dans des cellules résistantes au cis-platine [32]. Cependant, le rôle de cette protéine dans la réparation et la résistance reste encore à déterminer.

\section{Implications thérapeutiques de la connexion entre la réparation, la transcription et le cycle cellulaire}

Les connexions entre la réparation, la transcription et le cycle cellulaire ouvrent la voie à des alternatives multiples pour moduler la réparation dans le contexte thérapeutique (figure 3). Le traitement des cellules par les médicaments induit l'expression de toute une panoplie de gènes impliqués dans la réparation ainsi que celle de facteurs de transcription, de facteurs de croissance et de leurs récepteurs, et de protéines associées au stress et au processus inflammatoire (figure 3). La plupart des médicaments utilisés en chimiothérapie induisent l'activation de gènes associés à la réponse cellulaire immédiate aux lésions de l'ADN, en particulier les gènes Fos, Jun, p53, Gadd, polymérase $\beta$, $P K C$ (protéine kinase $\mathrm{C}$ ), les gènes codant pour les ubiquitines, ainsi que d'autres protéines nucléaires se liant aux adduits de l'ADN. Cette induction pourrait être liée, au moins en partie, à l'activation des récepteurs à activité tyrosine kinases, des MAP kinases $(m i-$ togen-activated protein kinases), protéine kinases associées à l'ADN. Ces dernières, activées par les lésions d'ADN, phosphorylent un certain nombre de protéines incluant Fos, Jun, la protéine de réplication HSSB (human singlestranded DNA-binding protein), et la p53 [33]. Bien que le rôle de cette in- il est fort probable qu'elle puisse contribuer à la régulation/activation des protéines de réparation et/ou à la modulation du cycle cellulaire à la suite des lésions de l'ADN. Dans ce dernier cas, la p53 est requise pour l'arrêt des cellules en G1, étape nécessaire pour la réparation $[34,35]$. L'inactivation de la p53 par des mutations est très fréquente dans les cancers humains et y est souvent associée à la résistance et aux rechutes. Les mutations dans la p53 sont également capables d'induire la résistance cellulaire aux facteurs de croissance [36] et de moduler les gènes de résistance. Ainsi il a été démontré que la p53 mutée stimule le promoteur du gène MDR1 [37]. L'association de la p53 à XPD/ERCC2 et XPB/ERCC3 suggère qu'elle est impliquée directement dans la régulation de NER [20].

Par ailleurs, des médicaments anticancéreux tels que l'adriamycine et le cisplatine activent le récepteur EGFR (epidermal growth factor receptor), les PKC et le phosphatidyl-inositol. Cette réponse souligne le rôle des signaux de transduction dans la réponse cellulaire aux lésions de l'ADN mais aussi suggère que ces voies pourraient être empruntées par les médicaments. La surexpression des gènes codant pour les récepteurs à activité tyrosine kinase comme c-ErbB-2 et EGFR, à la suite d'une amplification, d'un réarrangement ou de mutations, est souvent associée à une faible réponse cellulaire aux médicaments et à un pronostic clinique sévère. Nous avons trouvé une bonne corrélation entre le niveau de la protéine p185, produit du gène c-erbB-2, le niveau de réparation et la résistance des cellules pulmonaires aux analogues du cis-platine (résultats non publiés). D'autres études réalisées sur des cellules du cancer du sein ont démontré que la modulation du récepteur c-erbB-2 par des anticorps dirigés contre le domaine extracellulaire augmente la réponse cellulaire aux médicaments par un mécanisme qui semble impliquer la modulation de la réparation [38]. Tous ces exemples démontrent que les connexions entre la réparation, la transcription et le cycle cellulaire (figure 3) peuvent offrir des cibles multiples et potentielles pour manipuler la réparation dans le contexte de la modulation pharmacologique des médicaments anticancéreux.

\section{Conclusion}

Les données récentes ont montré que les protéines de réparation jouent des rôles multiples dans le processus physiologique de la cellule y compris la transcription et la régulation du cycle cellulaire. A l'exception de la protéine MGMT, le rôle de la réparation dans la résistance tumorale reste encore descriptif et des études plus approfondies sont nécessaires afin de mieux comprendre la fonction des différentes protéines impliquées ainsi que leurs voies de régulation. Il est également important de tenir compte de la complexité des mécanismes d'action des médicaments. Outre des lésions directes causées au niveau de l'ADN, de nombreux agents génotoxiques agissent par interaction avec les facteurs de transcription, par inhibition directe, antagonisme, ou en altérant les interactions protéine-protéine nécessaires à de nombreuses fonctions de régulation de gènes [39]. La connaissance de ces interactions est primordiale pour mieux comprendre les mécanismes de défense cellulaire et leurs implications thérapeutiques

\section{Remerciements}

Nous remercions tous les membres de nos laboratoires pour leurs contributions scientifiques. Les travaux mentionnés dans cette revue ont bénéficié du soutien financier du Conseil Médical de Recherches du Canada, de l'Association Pulmonaire du Québec et du Fond de Recherches en Santé du Québec « Réseau de Pharmacologie du Cancer» (M. A.-J.), et des subventions CA31721 et CA53791 de l'USPHS-USA (S. M.).

\section{TIRÉS À PART}

M. Alaoui-Jamali. 


\section{Summary}

DNA repair and its regulatory pathways in cancer pharmacology

A major limitation in the treatment of human cancer is the frequent development of resistance to chemotherapy drugs, that may occur either de novo or acquired after drug exposure. Tumor cells cope with DNA damage by a variety of mechanisms including activation of DNA repair processes and delay or arrest of cell-cycle progression. Dysregulation of DNA repair mechanisms has been shown to contribute to drug resistance in human cancer cells maintained either in vitro or in vivo. The $\mathrm{O}^{6}$-methylguanine-DNA methyltransferase (MGMT) is a well characterized protein which is responsible for the removal of alkyl groups from DNA bases, mainly $\mathrm{O}^{6}$ alkylguanine. Its role in tumor drug resistance has brought potential applications for DNA repair in cancer therapy ranging from biochemical modulation, aimed at reducing MGMT expression in tumor cells, to gene therapy approaches aimed at increasing MGMT expression in bone marrow cells and therefore protecting against myelosuppression side effects of drugs such as chloronitrosoureas. Drug resistance has also been associated with abnormalities in DNA nucleotide excision repair pathway in a variety of human tumor cell lines originated from ovarian adenocarcinomas, chronic lymphocytic leukemia, breast and lung cancers. However, the molecular basis of these abnormalities and their clinical relevance are still unknown. The inter-relationship between DNA repair, transcription, and several other processes that regulate the cell cycle indicates that checkpoint mechanisms are associated intimately with the DNA repair machinery. Numerous cell signaling pathways can also be triggered by DNA damage suggesting a role of cell signal transduction in the regulation of cellular response to DNA damage and DNA repair. The extraordinary progress in cloning genes involved in the regulation of DNA repair and cell cycle provides tools to begin to unfold the role of these pathways in cell response to external stress. It is likely that an understanding of these mechanisms may offer novel targets for cancer therapeutics. 